\title{
Design and Test with a Tomato Identification System based on Visual Technologies
}

\author{
Ya-dong Tang , Jing-zhao Shi , Yong-chang Yu*, Yu-jing He , He Li and \\ Shuai-jun Zhang
}

The Agriculture University of Henan, College of Machinery and Electronic Engineering, Zhengzhou, China, 450002

Keywords: BQPSO Algorithm, Threshold Segmentation, Offset Extract, Characteristic Matching.

\begin{abstract}
In this paper, a boundary control-based algorithm Binary Quantum Particle Swarm Optimization (BQPSO) that considers quantum particle swarm with Delta potential well was used to determine otsu threshold. In the optimization, particles moved in the Delta potential well with the best position POPSIZE as center. The best threshold was determined by updating individual extremum of a single particle pbest and global extremum of particles swarm gbest to their good-enough fitness values, in order for image segmentation. As for profiles, random circle method was used to extract radius of fruit circle. With binocular vision system, a Fourier-transform algorithm was adopted to extract offsets of left and right tomato images, and by marking their sorting baseline, they were matched according to sequential consistency principle.
\end{abstract}

\section{Introduction}

With natural light source, accurate identification and shape-feature detection of ripe tomatoes in complex background lay foundation of the visual system of tomato harvesting robots and their coordinative operations [1-3]. Reference [2] performed statistical analysis with color indicators of targeted tomato images and their backgrounds in different colored spaces, to determine characteristic color component of harvesting tomatoes; then with different segmentation algorithms, comparison of segmentation results was carried out, in order to build an improved tomato identification model with Ostu segmentation algorithm, but showing imperfect timeliness. Reference [3] proposed a Fuzzy Clustering Means (FCM) algorithm for fuzzy clustering, and by summing the clustered fruit images and partial lost targeted images, performed image segmentation of targeted tomato images, but showing a precision to be improved further.

In this paper with a boundary control-based BQPSO algorithm, the Otsu threshold was determined in order for image segmentation of ripe tomatoes and their identification. Then a random circle method was used to extract characteristic shape component of tomato images. With binocular vision system, Fourier transform was carried out to extract offsets of tomato images, and by marking their sorting baseline, they were matched according to sequential consistency principle.

\section{Tomato Identification and Image Pretreatment}

\section{Fruit Identification}

Image segmentation is a key step to identify a fruit. Y. Q. Wang et al performed adaptive Ostu threshold segmentation by using images synthesized with $2 \mathrm{R}-\mathrm{G}-\mathrm{B}$ in RGB color space. This method had disadvantages of easily being affected by backlight and shadow, and many red color components in the background were difficult to be segmented [4]. Traditionally Otsu threshold is calculated by searching the best threshold combination in full gray scale. Considering the Otsu threshold calculation is time-consuming, the algorithm Quantum Particle Swarm Optimization (QPSO) is introduced in the field of image threshold segmentation, to reduce an engineering issue to an optimization issue. Potentially this results in an issue of exploring the best position of spatial particle travel. By determining the Otsu threshold with a coordinative and compression-factored improved particle swarm algorithm, the threshold calculation time period is reduced significantly, 
facilitating improvement of image treatment timeliness. However, the algorithm QPSO shows poor performance when the best solution just exists on the boundary [5].

In this paper, a boundary control-based algorithm BQPSO was used for image segmentation of tomato from its background by fitness evaluation of particles with the maximum inter-class variance ratio of targeted tomatoes and their background as a fitness function. Also, boundary control theory was introduced in this paper. In the optimization of particles, when flying out of the search boundary, they were relocated to some point near the boundary of the search zone by changing their position in a random manner. This avoided search of locally optimal solution and increased the particle swarm diversity, improving global search capacity of the algorithm [6].

As shown in Figure 1, there is much red color of ripe tomatoes $(\mathrm{R})$ and green color of the background $(\mathrm{G})$ in the treated ripe tomatoes image. $R, G$ are significantly different in gray value. Assume that the image of harvested tomatoes $\mathrm{F}(\mathrm{x}, \mathrm{y})$ has gray level of $(0,1,2, \ldots, \mathrm{L}-1)$, and its neighboring smoothing image $\mathrm{g}(\mathrm{x}, \mathrm{y})$ that uses $3 \times 3$ neighboring average value of a pixel as gray value also has gray level of $\mathrm{L}$ (representing degree of convergence of the particle swarm). Each pixel in the image has a binary group consisting of: gray value of pixel and its neighboring average value. Assume that the pixel gray value is $i$, the number of pixels involved in calculation of neighboring average value is $j$, and the total number of pixels in the image is $\mathrm{M}$. From the line profile, any given threshold $\mathrm{t}$ can be used to segment the image into two classes of targeted tomatoes and their background.

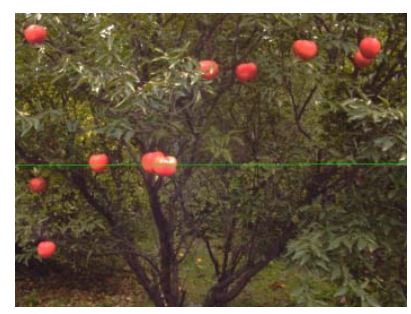

(a) Original image

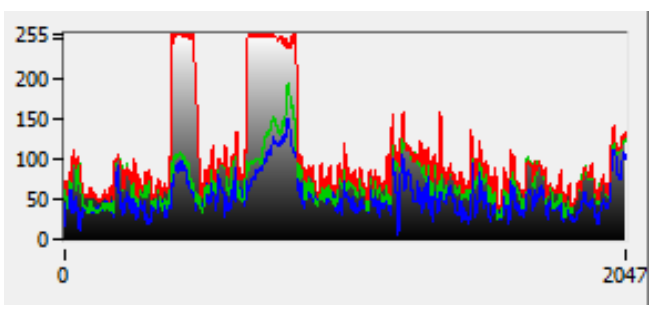

(b) Line profile

Figure 1. DNRG image

POPSIZE particles are created randomly between $(0,255)$, each of which has DIMENSION dimensions. The creation position of particles is taken as initial value in the course of searching the best position in their travel, and the maximum number of iterations is set as MAXITER. Assume that a tomato and its background are respectively $\mathrm{C} 1$ and $\mathrm{C} 2$, with their occurrence probabilities expressed as follows:

$$
\begin{gathered}
\omega_{1}=\sum_{i=1}^{r-1} \sum_{j=1}^{g-1} P_{i j} \\
\omega_{2}=\sum_{i=1}^{\mathrm{r}-1} \sum_{j-1}^{g-1} P_{i j}
\end{gathered}
$$

Their corresponding mean vectors are:

$$
\begin{gathered}
\mu_{1}=\left(\mu_{1 \mathrm{i}}, u_{1 j}\right) T=\left(\sum_{i=1}^{r-1} \sum_{j=1}^{g-1} i P_{i j}, \sum_{i=1}^{r-1} \sum_{j=1}^{g-1} j P_{i j}\right) \\
\mu_{2}=\left(\mu_{2 i}, \mu_{2 j}\right) T=\left(\sum_{i=1}^{L-1} \sum_{j=1}^{L-1} i P_{i j}, \sum_{i=1}^{L-1} \sum_{j=1}^{L-1} j P_{i j}\right)
\end{gathered}
$$


The mean vector of the two-dimensional histogram is:

$$
\mu=\left(\mu_{1}, \mu_{2}\right)^{T}=\left(\sum_{i=0}^{L-1} i P_{i j}, \sum_{i=0}^{L-1} j P_{i j}\right)
$$

By segmenting the image with threshold $t$, the intra-class variance is:

$$
\sigma_{\mathrm{w}}^{2}=\omega_{1} \sigma_{1}^{2}+\omega_{2} \sigma_{2}^{2}
$$

The inter-class variance is:

$$
\sigma_{B}^{2}=\omega_{1}\left(\mu_{1}-\mu_{T}\right)_{2}+\omega_{2}\left(\mu_{2}-\mu_{T}\right)^{2}
$$

The maximum inter-class variance ratio is:

$$
\eta_{\mathrm{t}}=\operatorname{MAX}\left\{\frac{\sigma_{B}^{2}}{{\sigma_{W}}^{2}}\right\}
$$

Fitness evaluation of particles is carried out with the maximum inter-class variance ratio as a fitness function. To avoid random placement of particles in the search zone and destruction of particle swarm stability, after introducing the boundary control strategy, out-of-boundary particles do not concentrate on the boundary in the optimization of image threshold, but return to near search zone.

Update the position of particles according to Equation (8) and check whether the ending condition is met or not (meeting well-enough fitness values or MAXITER), and if not, proceed with the next step. Let $\mathrm{t}=\mathrm{t}+1$, go back to Step 2 until gbest(i)=MAXITER.

Update individual extremum of each particle Pbest(i) (i=0, 2, 3,..,POPSIZE-1) and global extremum of the whole particle swarm gbest(i) $(i=0,2,3, \ldots$, POPSIZE- 1$)$. With evolution of the particle no. $i$ from $t$ into $t+1$, it is updated with the following equations:

$$
\begin{array}{r}
\left\{\begin{array}{l}
X_{\text {id }}(\mathrm{t}+1)=P_{\text {id }}-\beta \cdot \mid \text { mbest }_{\mathrm{d}}-X_{\text {id }}(t) \mid \cdot \ln (1 / \mu), \mu>0.5 \\
X_{\text {id }}(\mathrm{t}+1)=\mathrm{P}_{\text {id }}-\beta \cdot \mid \text { mbest }_{\mathrm{d}}-X_{\text {id }}(t) \mid \cdot \ln (1 / \mu), \mu \leq 0.5
\end{array}\right. \\
P_{\text {id }}=\left(\delta_{1} \cdot P_{\text {id }}+\delta_{2} \cdot P_{\text {gd }}\right) /\left(\delta_{1}-\delta_{2}\right) \\
\text { mbest }=\frac{1}{M} \sum_{i=1}^{M} P_{i}=\left(\frac{1}{M} \sum_{i=1}^{M} P_{i 1}, \frac{1}{M} \sum_{i=1}^{M} P_{i 2}, \ldots \ldots, \frac{1}{M} \sum_{i=1}^{M} P_{i d}\right)
\end{array}
$$

Where in Xid is particle position; Pid is pbest of the particle no. $\mathrm{i} ; \quad \beta$ is contraction-expansion factor; $\mu, \delta_{1}$ and $\delta_{2}$ are random numbers distributed evenly in $[0,1]$; Pgd is pbest of the particle swarm, mbest is the average best position of all particles in the swarm; and $M$ is the number of particles.

Considering spatial features of the image, when treating the image with the algorithm BQPSO, the boundary control strategy is introduced in order to improve global search capacity of threshold 
segmentation. By changing particle position in a random manner, the particle is relocated to some point near the boundary of the search zone, with the boundary control equation shown as follows:

$$
X_{i d}= \begin{cases}X_{\min }+c \cdot\left(\operatorname{rand}() \%\left(X_{\max }-X_{\min }\right)\right. & , X_{\mathrm{id}}<X_{\min } \\ X_{\max }-c \cdot\left(\operatorname{rand}() \%\left(X_{\max }-X_{\text {min }}\right)\right) & , X_{\mathrm{id}}>X_{\text {min }}\end{cases}
$$

Wherein Xmin and Xmax refer to the minimum and maximum positions of particles respectively, and after being treated in the equation by adding an adjustment coefficient $\mathrm{c} \quad(0<\mathrm{c}<1)$, out-of-boundary particles will randomly appear near the boundary $\mathrm{c} \cdot\left(\operatorname{rand}() \%\left(X_{\max }-X_{\min }\right)\right.$.

Then image segmentation is completed by determining the position of the particle swarm, i.e. outputting the best threshold. From analysis of gray histogram of the image, few particles appear on the left and right sides. The segmented image is binarily treated as follows:

$$
\begin{aligned}
& \text { If } f(r, g)>\mathrm{r}^{*} \text { and } f(r, g)<\mathrm{g}^{*} \text { Then } f r *, g *(r, g)=1 \\
& \text { If } f(r, g)<\mathrm{r}^{*} \text { or } f(r, g)>\mathrm{g}^{*} \text { Then } f r *, g *(r, g)=0
\end{aligned}
$$

Gray values in the image are positive integers in $[0,255]$. However positions obtained from the BQPSO updating equation are continuous, and thus after each updating, the positions are reduced to integer values and checked whether out of boundary or not (greater than 255 or less than 0) [7].

\section{Image Pretreatment}

Noise removal, hole filling and smooth boundary treatment is carried out for image binarization segmented with the best threshold. With a NXN median filter, if the gray level of a pixel is output equal to or greater than that of its neighboring (N2-1)/2 pixels, and less than or equal to that of (N2-1)/2 pixels screened with the NXN median filter, then the gray level of such pixel is taken as the output gray level [8].

Since calculation with the median filter is relatively slow and its noise removal is dependent on two related but completely separate elements: neighboring spatial dimension and number of pixels involved in the median calculation. As for a large filter, its noise removal and increase of the number of pixels involved in the median calculation are non-linear, so a sparse median filter is used [9]. In sum, a cross median filter was used in this paper to eliminate random foreground points occurring after image binarization. Then each regional area was calculated and the region of having $1 / 8$ of the best area was removed to eliminate larger noise points [10]. Wavelet transform algorithm was used to improve weak edge of tomatoes adhesion, and Canny edge detection was carried out at low-frequency components. The higher threshold of Canny operator was determined from Ostu, and its lower threshold was equal to a half of the higher threshold. Finally the image edge was extracted.

\section{Extraction of Shape Feature from Tomatoes}

Tomato image shows irregular roundness, and its profile after image pretreatment is extracted for shape feature with random Hough method. As long as most of points in the tomato profile fall into a circle, it is believed that the circle represents the one within which the tomato profile is located [11]. Principle of circle determination: any three non-collinear points in the tomato profile are chosen to determine a circle with its radius $r$ and center position (Ox, Oy). Assume that the determined circle has a width $\mathrm{w}$, and any point in the tomato profile falls into the circle. Then coordinates of the point $(\mathrm{x}, \mathrm{y})$ shall meet:

$$
\mathrm{r}-\frac{w}{2} \leq \sqrt{\left(x-O_{x}\right)^{2}+\left(y-O_{y}\right)^{2}} \leq r+\frac{w}{2}
$$


The random circle algorithm has flow chart as shown in Fig. 2:

N

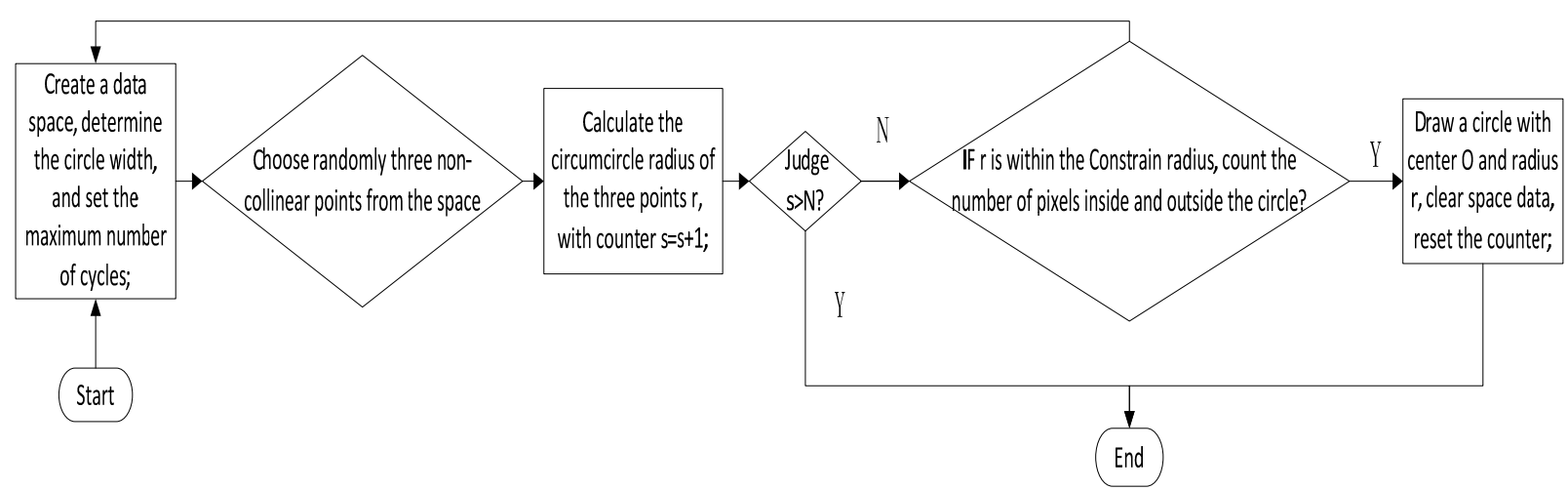

Figure 2 Block diagram of random circle algorithm

The random circle algorithm (RCR) provides good circle extraction for neighboring fruits or ones whose profiles are not shielded, but shows poor performance for fruits whose profiles are significantly shielded or obviously different from a circle. However this disadvantage can be compensated from diversion of camera angle. With the method above, circle images of tomatoes are extracted as shown Fig. 3.

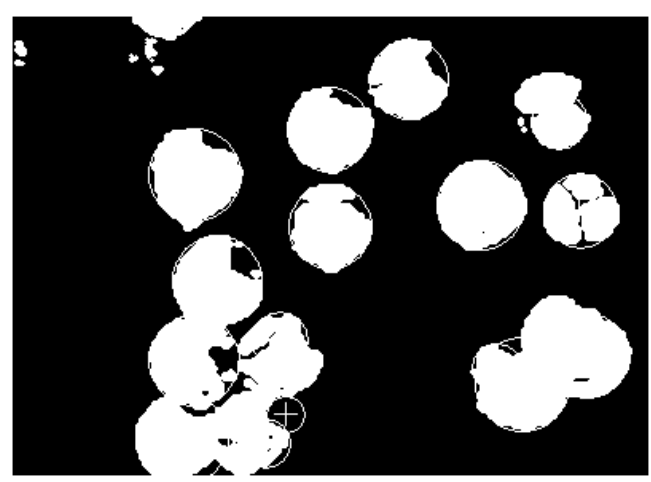

Figure 3 Result of circles extraction

\section{Test and Results}

After overall construction of the system, a test is required to verify its accuracy and detection precision, in order for better performance in applications.

\section{Experimental material}

The tomato image acquisition system used in this paper consists of two CMOS color cameras (MV-V300 model) with $80 \mathrm{~mm}$-focus camera lens, between which the baseline length is $20 \mathrm{~cm}$. Image acquisitions are sourced from growth state simulation test of tomatoes in the campus of Henan Agricultural University, and the sample tomato is a TY virus resistive species. The image treatment software is labview2015.

\section{Results and Analysis}

In this paper the system was verified by two tests of comparison with different image segmentation algorithms and of fruit extraction and matching. 


\section{Comparison with Different Image Segmentation Algorithms}

Image pretreatments of tomatoes were carried out respectively with algorithms of 2R-G-B, QPSO and BQPSO, and after contrast enhancement, these images were shown in Fig. 4(c).
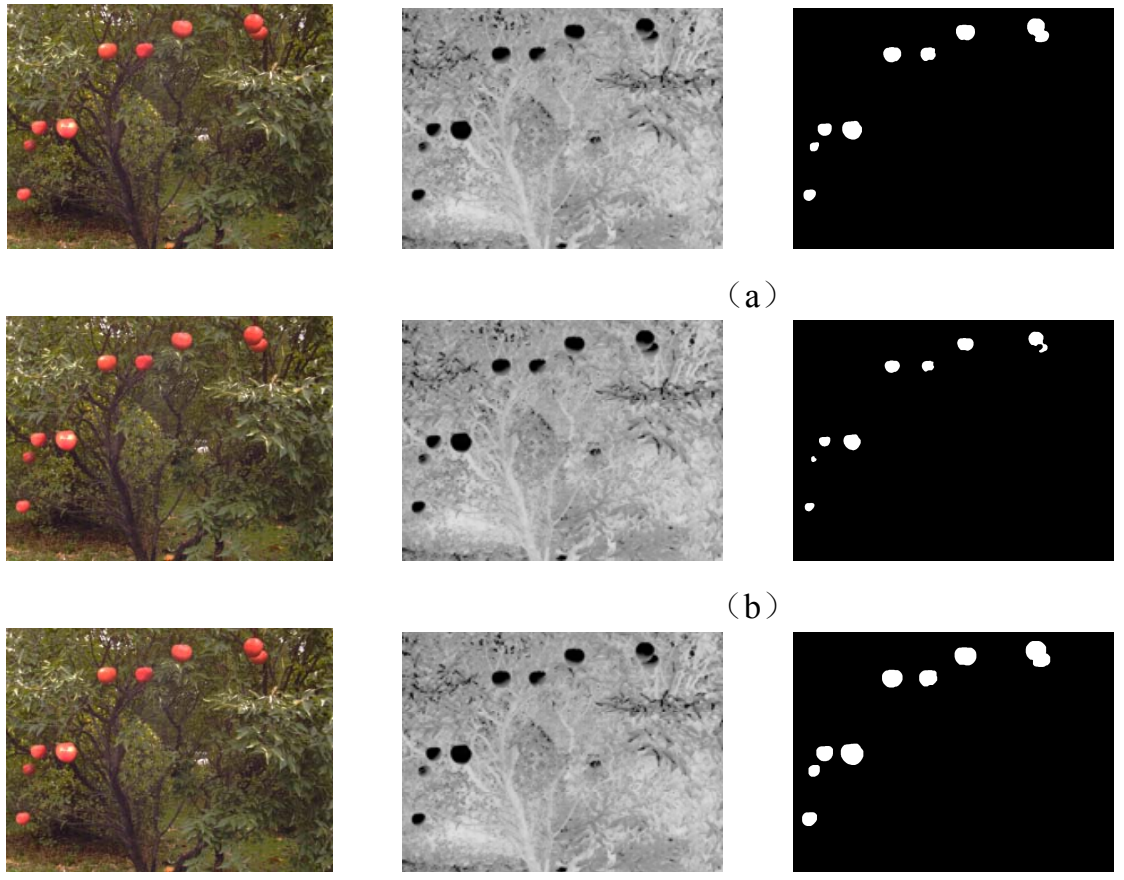

(b)

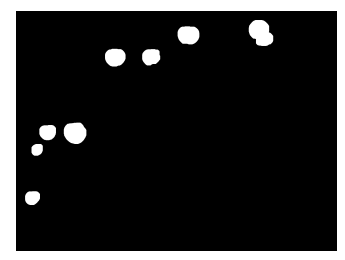

(c)
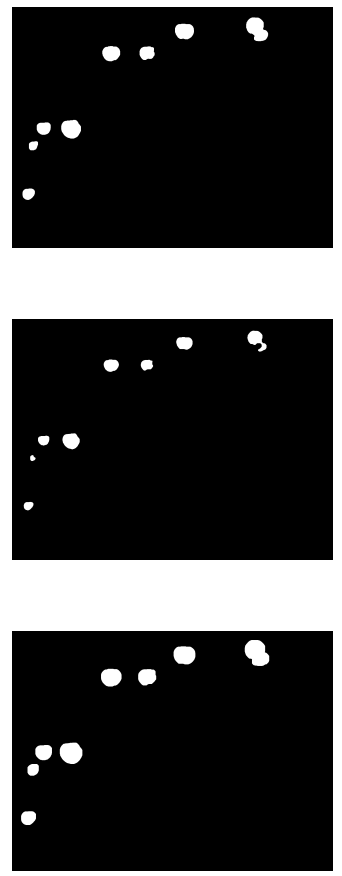

Figure 4 Comparison of image segmentation results
(a) 2R-G-B
(b) QPSO
(c) BQPSO

With 100 image acquisitions of tomatoes, the three algorithms above were used for image segmentation, with segmentation error rates shown in Fig. 5. Obviously the first two algorithms show higher segmentation error rates than BQPSO used in this paper.

With the improved algorithm BQPSO, image segmentation with Ostu threshold and image pretreatment were carried out for these 100 acquired images, which contained 863 tomatoes (with their shielding area less than $1 / 3$ of tomatoes). Then 803 tomatoes were identified, with successful identification rate equal to $93.1 \%$. With natural light source, the boundary control-based algorithm BQPSO used for Ostu image segmentation overcome adverse factors of backlight and bare soil to some extent. The identification test of 100 tomato images showed that, the system provided good identification for ripe tomatoes. The algorithm BQPSO showed stronger robustness and reliability in optimization result, operation efficiency and stability than 2R-G-B and QPSO. Error identification was caused as result of two factors: dense branches and leaves in partial images resulted in dark light, also with backlight, and red color components in branches and leaves increased in the images; partial images were overexposed. 


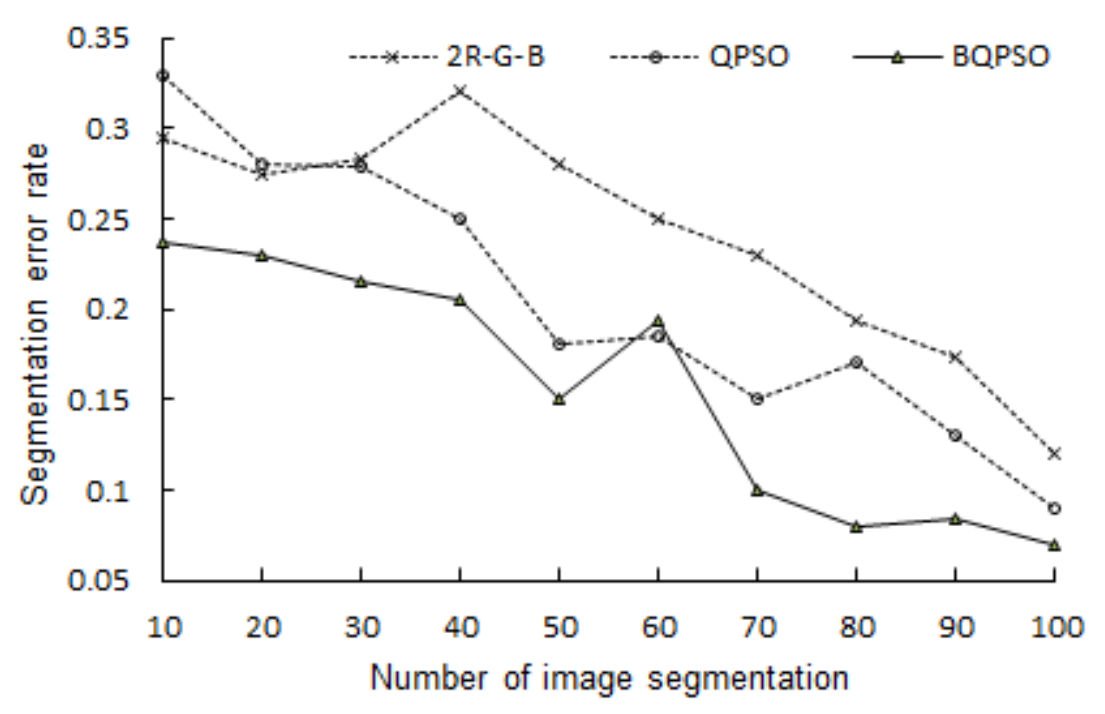

Figure 5 Segmentation error rate

\section{Feature Extraction from Fruits}

As for 100 images (using JPG format and containing $2048 \times 1536$ pixels for each image) acquired in different light conditions, circle center and radius of an identified tomato in the images were extracted with the random circle method (RCR), with circle width w containing 6 pixels, number of cycles set as 200, and radius restricted within 30-60 pixels. Extraction results were shown in Tab. 1.

Table 1. Extraction results with RCR

\begin{tabular}{ccccc}
\hline Images/nos & $\begin{array}{c}\text { Total } \\
\text { tomatoes/nos }\end{array}$ & $\begin{array}{c}\text { Effective } \\
\text { tomatoes/nos }\end{array}$ & $\begin{array}{c}\text { Correctly } \\
\text { extracted } \\
\text { tomatoes } / \text { nos }\end{array}$ & $\begin{array}{c}\text { Correct } \\
\text { extraction } \\
\text { rate } / \%\end{array}$ \\
\hline 100 & 863 & 818 & 779 & 95 \\
\hline
\end{tabular}

Then 42 images (21 left images and 21 right images) were tested for matching, with test results as shown in Tab. 2. Error matching was caused as result of poor fruit identification due to too much shielding in an image.

\section{Conclusions}

(1) The Ostu threshold segmentation with the boundary control-based algorithm BQPSO was carried out for identification of ripe tomatoes in natural light conditions. Test results showed that, the algorithm BQPSO provided effective identification of ripe fruits with its successful identification rate up to $93.1 \%$, generally meeting requirements of tomatoes harvesting. But in complex factor conditions of significantly varied lighting and over exposure, etc, tomato identification with the algorithm still needs to be further improved.

(2) The random circle method was used to extract characteristic shapes from fruits. Test results showed that this method provided effective extraction of characteristic shapes from fruits with its correct extraction rate up to $95 \%$. As for single and neighboring fruits whose profiles were partially shielded, the circle extraction with the random circle method was good, but the circle extraction for fruits significantly shielded or obviously different from a circle was poor. 


\section{Acknowledgement}

This research was financially supported by Chinese National Soybean Industrial Technology System.

\section{References}

[1] H. Li, K. Wang, and Q. Cao. Tomato targets extraction and matching based on computer vision [J]. Transactions of the Chinese Society of Agricultural Engineering, 2012,28(5):168-172.

[2] X. L. Lv, X. R. Lv, and B. F. Lu. Identification Methods of Picking Tomatoes Based on Color Information [J]. Computer Engineering, 2010,36(11):178-179.

[3] F. C. Wang, Y. Xu, and H. B. Song. Study on Identification of Tomatoes-Based on Fuzzy Clustering Algorithm [J]. Journal of Agricultural Mechanization Research, 2015,(10):24-28.

[4] Y. Q. Wang, and H. Gao. Study on the Segmentation and Orientation of Fruit Image Under Natural Environment [J]. Computer Engineering, 2004,30(13):128-129.

[5] X. N. Tong. Dual image threshold segmentation based on quantum particle swarm algorithm [J]. Journal of Sichuan University, 2010,26(3):42-49.

[6] Sun J, Feng B, Xu W B. Particle Swarm Optimization with ParticlesHaving Quantum Behavior[C]. Proceedings of 2004 Congress on EvolutionaryComputation, 2004: 325-331.

[7] M. J. Zhang, X. Li, and Y. J. Wang. Underwater color image segmentation based on weight adjustment for color-to-gray [J]. Journal of Harbin Engineering University, 2015,(5):707-713.

[8] H. J. Wang, N. Shi, and H. Zhao. Image Preprocessing Technology Based on Improved Median Filter [J]. Computer Systems \& Applications, 2015,(5):237-240.

[9] H. Yao. New Combination Filtering Algorithm Based on Salt \& Pepper Noise in Digital Image [J]. Video Engineering, 2014, 38(1), 39-42.

[10] J. Y. Shi, and S. D. Liu. Dynamic window-based adaptive median filter algorithm [J]. Computer Engineering and Design. 2011, 32(9):3115-3118.

[11] Takita K, Aoki T, Sasaki Y, et al. High-Accuracy Subpixel ImageRegistration Based on Phase-Only Correlation. IEICE Trans on Fun-damentals of Electronics, Communications and Computer Sciences,2003, E86-A(8): 1925-1934. 